Supporting Information

\title{
Hyper-crosslinked polymers-derived nitrogen-doped hierarchical porous carbon as metal-free electrocatalysts for high-efficient oxygen reduction
}

Yue Yang, Zhuopei He, Shaolei Wang*, Heng-guo Wang*, and Guangshan Zhu

Key Laboratory of Polyoxometalate and Reticular Material Chemistry of Ministry of

Education, Faculty of Chemistry, Northeast Normal University, Changchun, 130024, China

*E-mail: wangs1030@nenu.edu.cn;

*E-mail:wanghg061@,nenu.edu.cn 


\section{Supplementary figures}
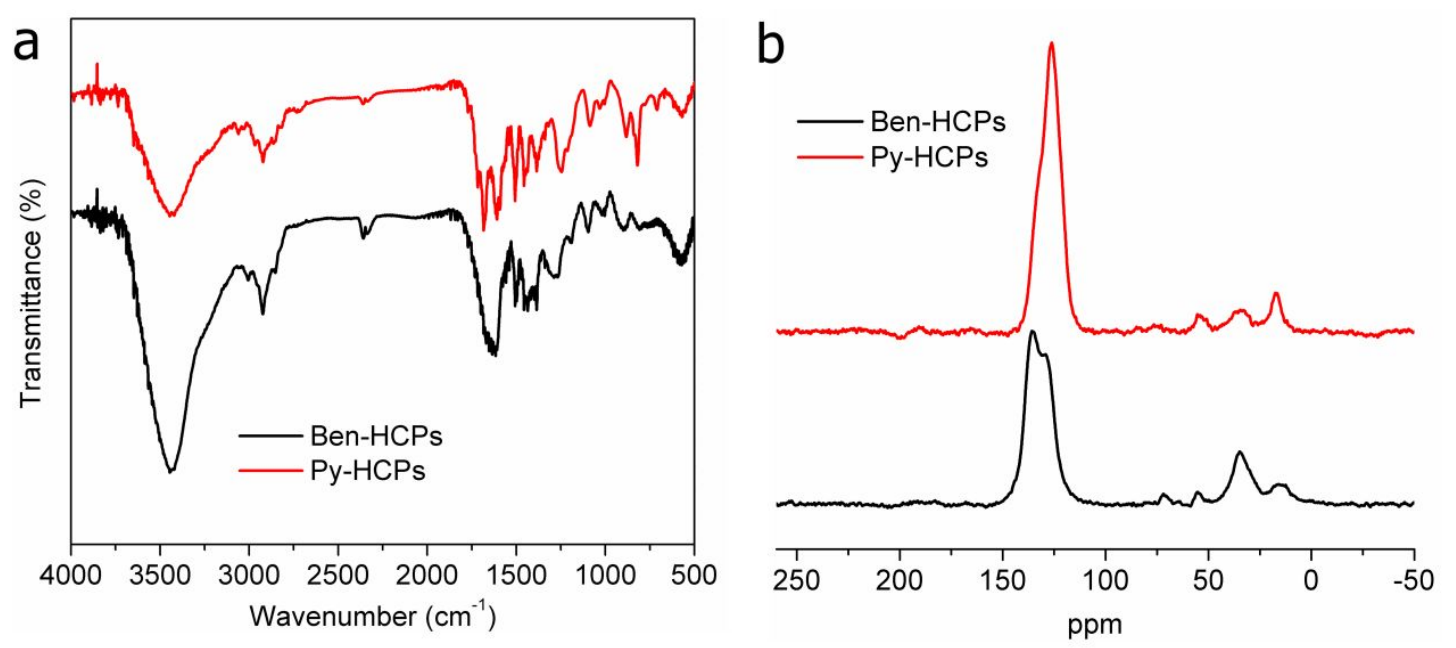

Figure S1. (a) The FT-IR spectra and (b) solid state ${ }^{13} \mathrm{C}$ cross-polarization magic-angle spinning NMR spectra of Ben-HCPs and Py-HCPs.

In the FT-IR spectra of Ben-HCPs and Py-HCPs, peaks near 1600, 1500 and $1450 \mathrm{~cm}^{-1}$ are attributed to aromatic ring skeleton vibrations, which are consistent with the structure of monomers. Meanwhile, the strong C-H stretching vibrations near $2920 \mathrm{~cm}^{-1}$ is attributed to the alkyl groups, suggesting that the networks of the resulting polymers contain abundant alkyl groups. The ${ }^{13} \mathrm{C}$ CP/MAS NMR of all samples shows resonance peaks near 137 and $130 \mathrm{ppm}$ due to substituted aromatic carbon and nonsubstituted aromatic carbon respectively, and the resonance peaks near $36 \mathrm{ppm}$ from carbon in methylene linker formed after Friedel Crafts reaction. 

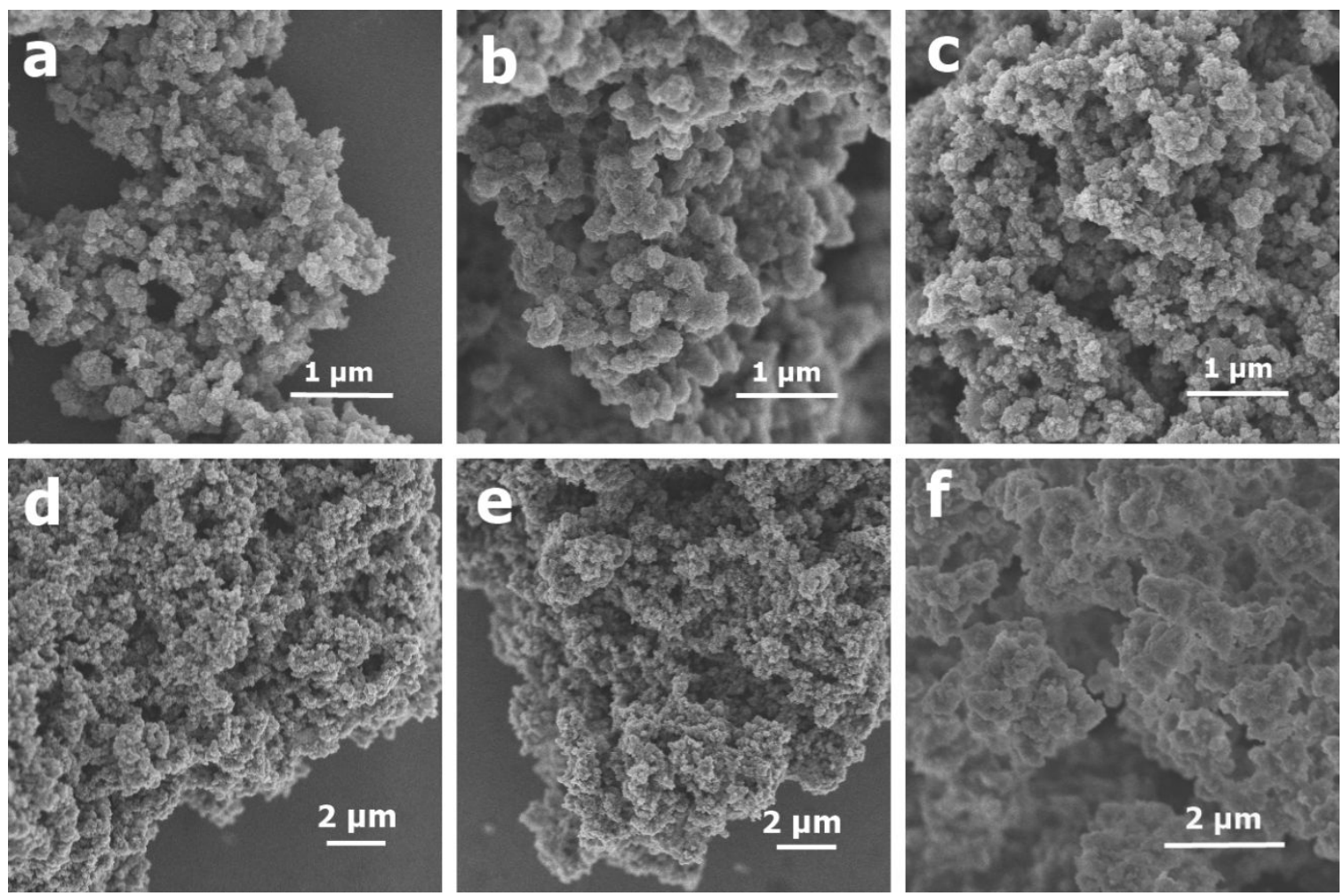

Figure S2. SEM images of (a) Ben-HCP-800, (b) Ben-HCP-900, (c) Ben-HCP-1000, (d) Ben-NHCP-800 (e) Ben-NHCP-900 and (f) Ben-HCPs. 

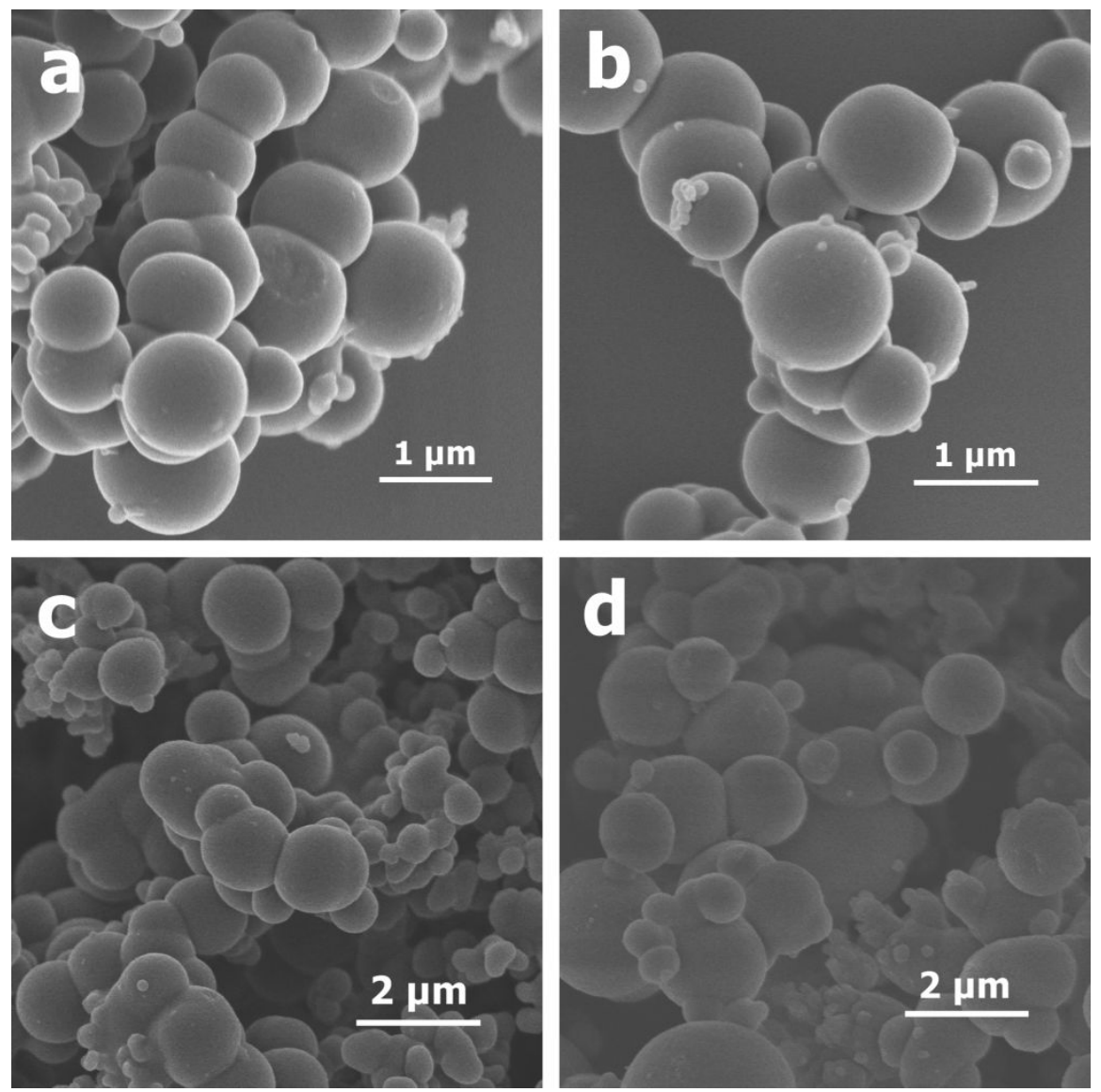

Figure S3. SEM images of (a) Py-HCP-900, (b) Py-HCP-1000, (c) Py-NHCP-900 and (d) Py-HCPs. 

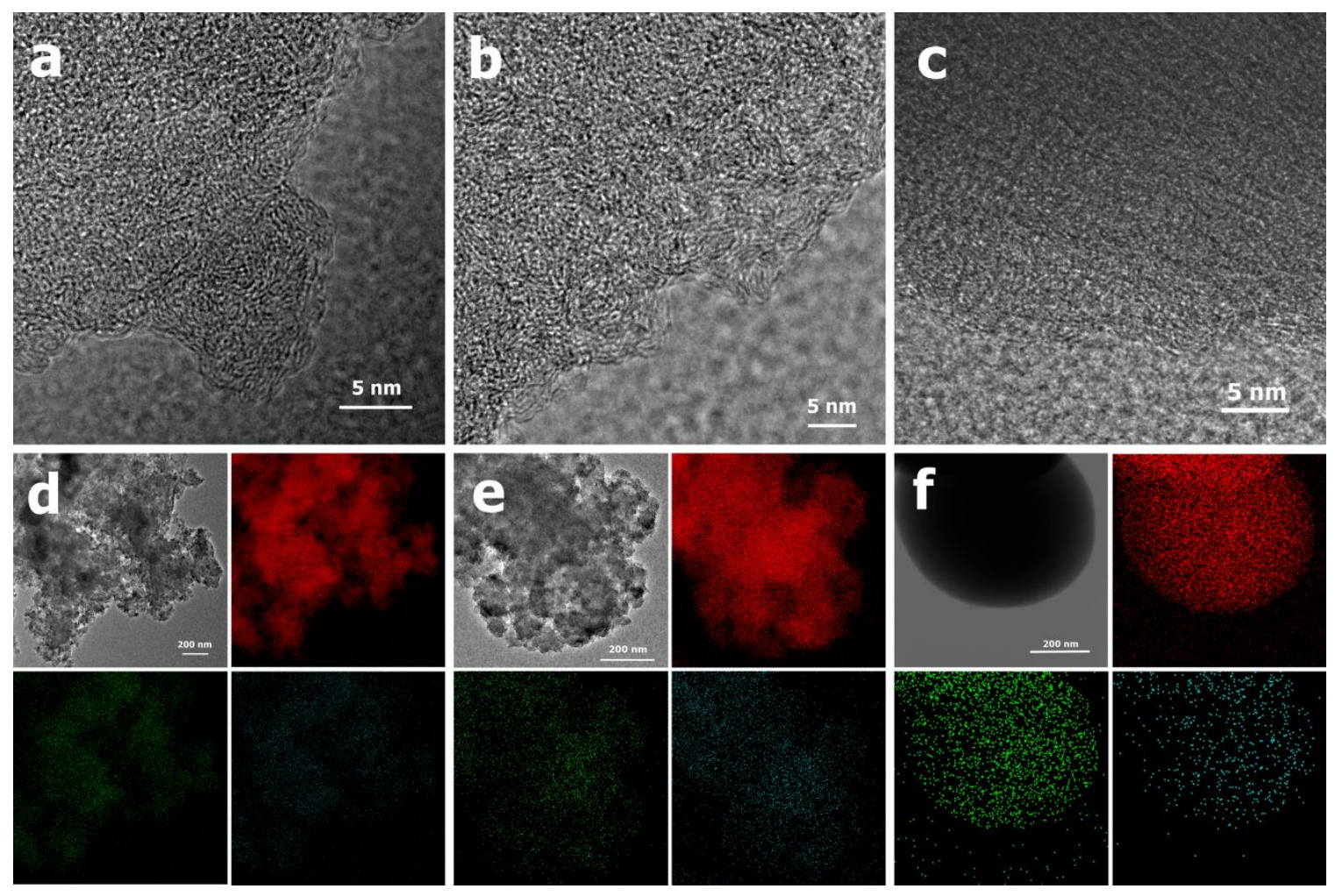

Figure S4. HR-TEM images of (a) Ben-NHCP-800, (b) Ben-NHCP-900 and (c) Py-NHCP-900; TEM elemental mapping of (d) Ben-NHCP-800, (e) Ben-NHCP-900 and (f) Py-NHCP-900. 

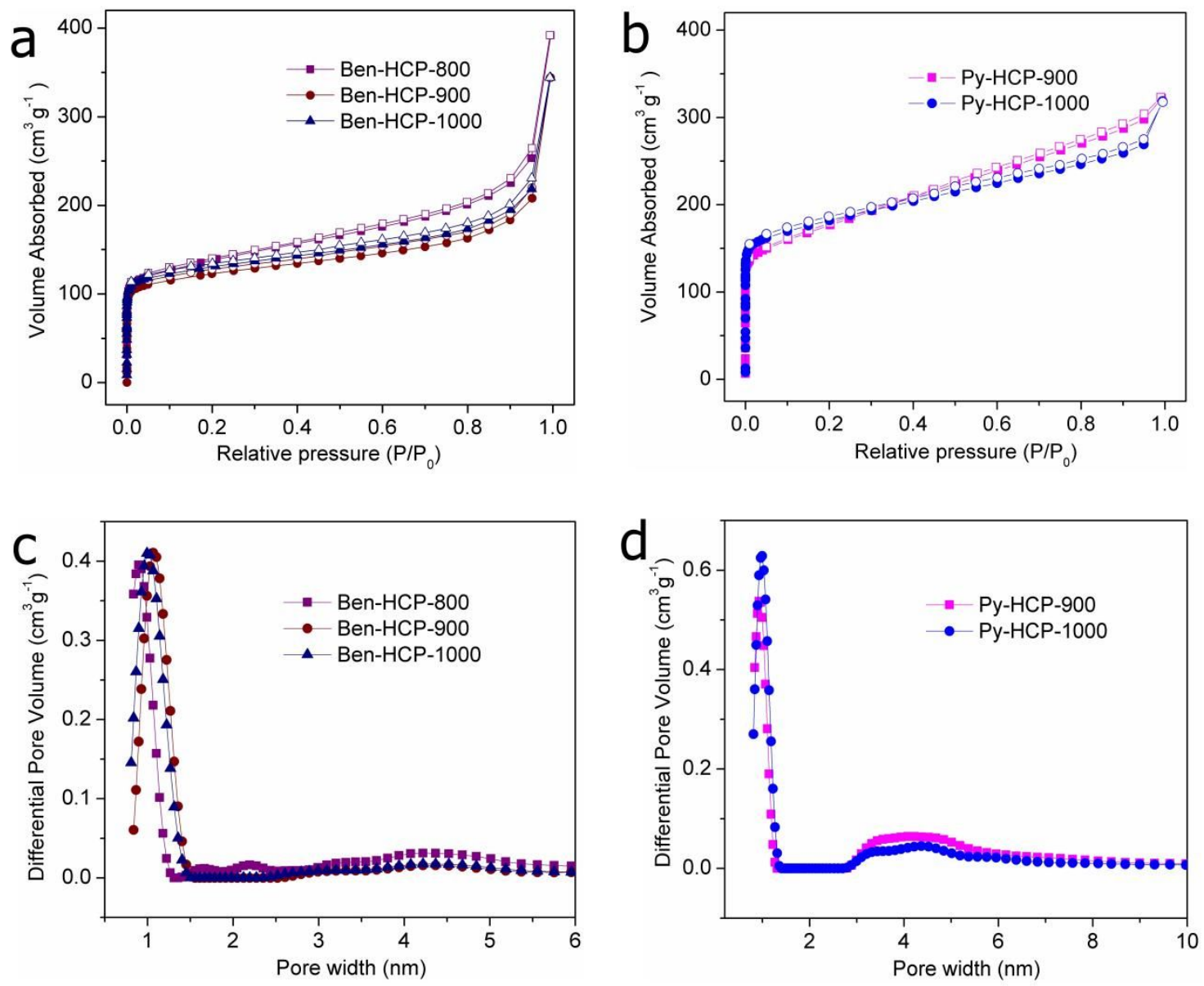

Figure S5. $\mathrm{N}_{2}$ absorption/desorption isotherms of (a) Ben-HCP-x and (b) Py-HCP-x; the corresponding pore size distribution of (c) Ben-HCP-x and (d) Py-HCP-x. 

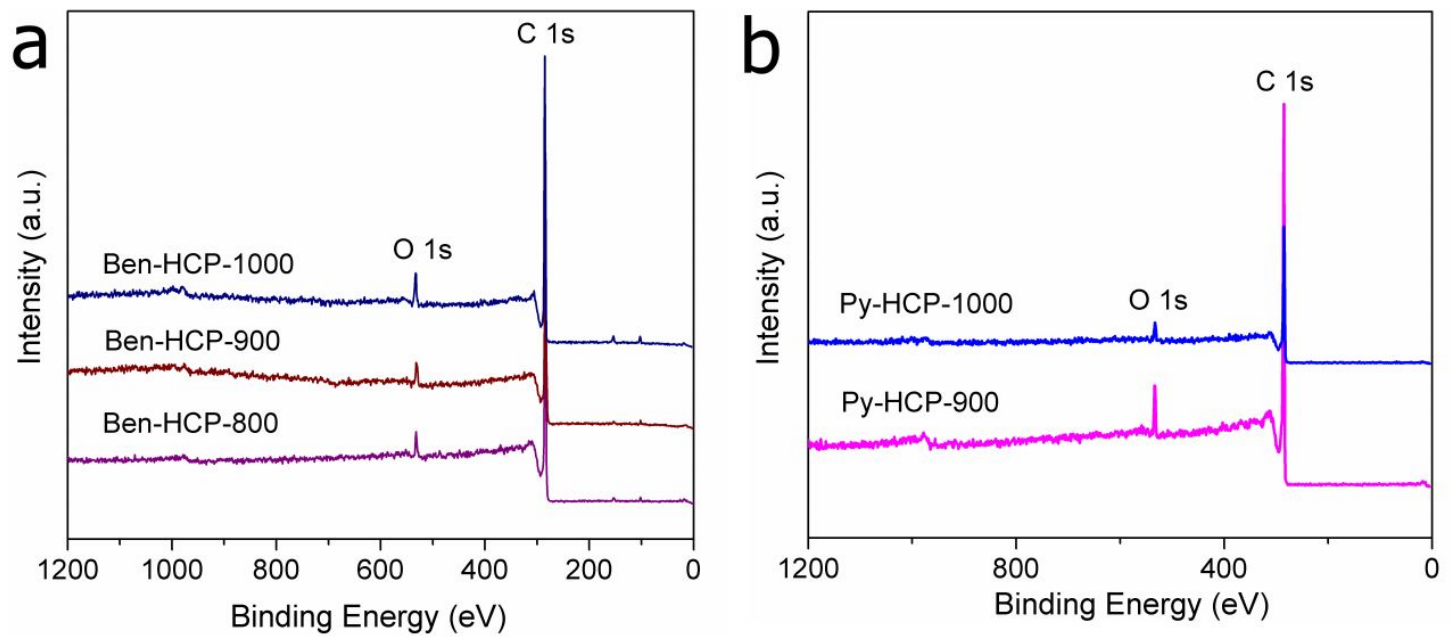

Figure S6. XPS spectra of (a) Ben-HCP-x and (b) Py-HCP-x. 

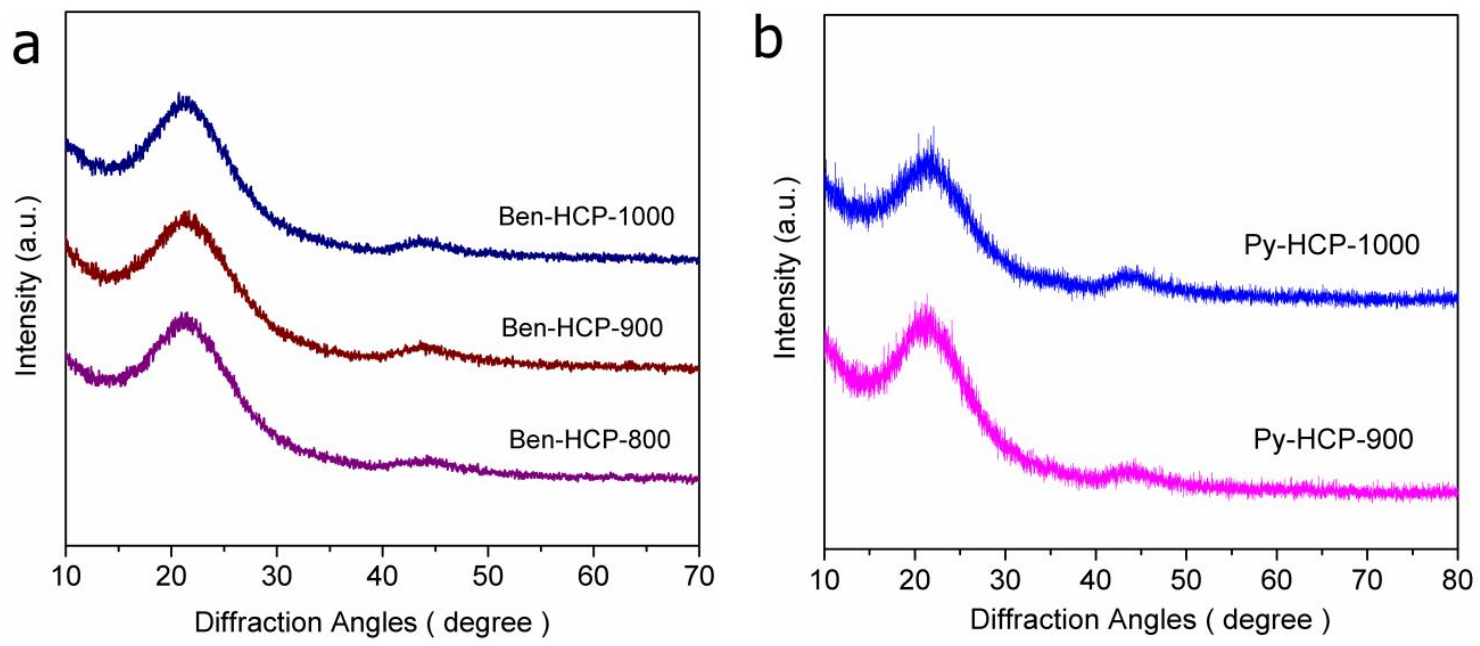

Figure S7. XRD patterns of (a) Ben-HCP-x and (b) Py-HCP-x. 

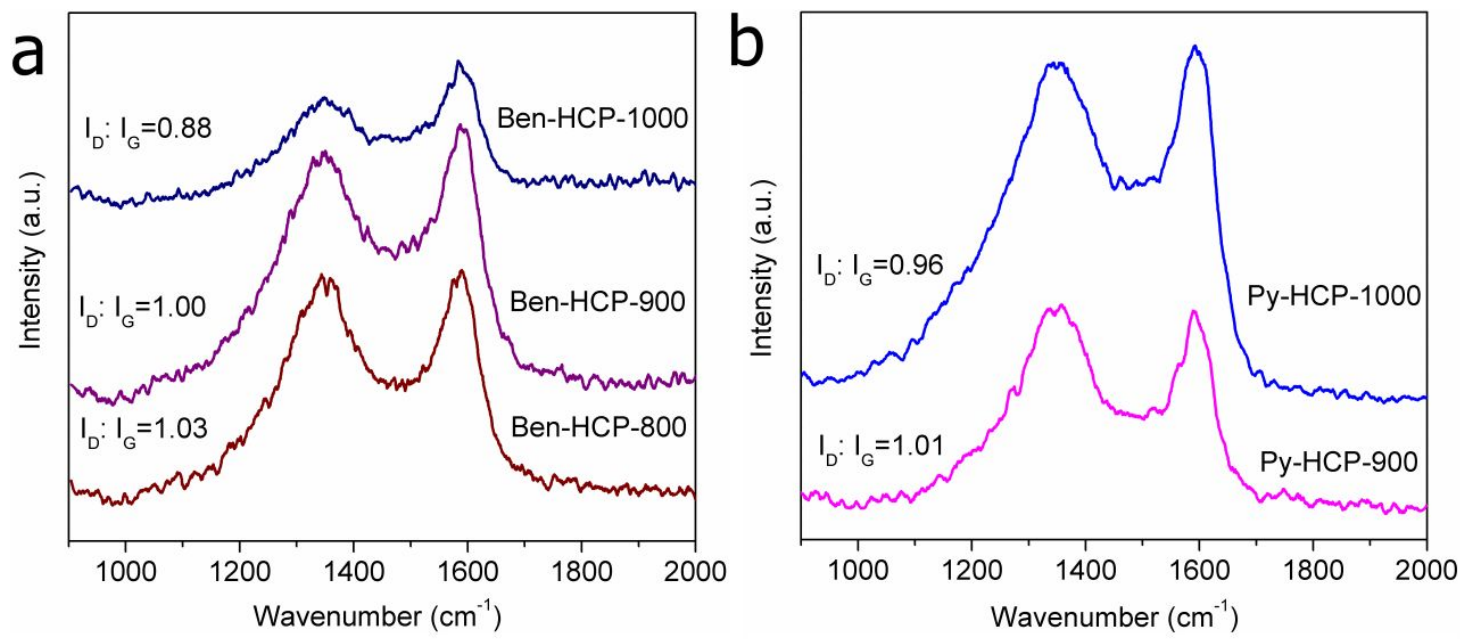

Figure S8. Raman spectra of (a) Ben-HCP-x and (b) Py-HCP-x. 

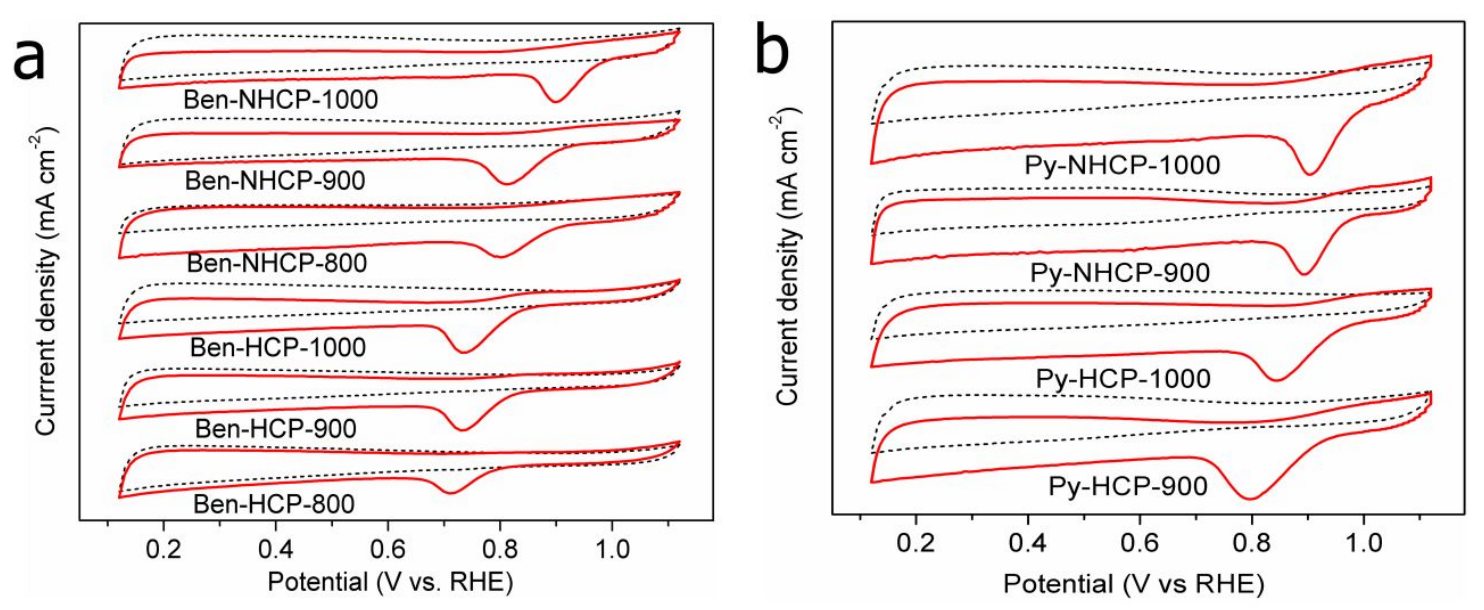

Figure S9. Cyclic voltammograms curves of (a) Ben-HCP-x, Ben-NHCP-x and (b) Py-HCP-x, Py-NHCP-x in $\mathrm{N}_{2-}$ and $\mathrm{O}_{2}$-saturated 0.1 M KOH electrolyte. 

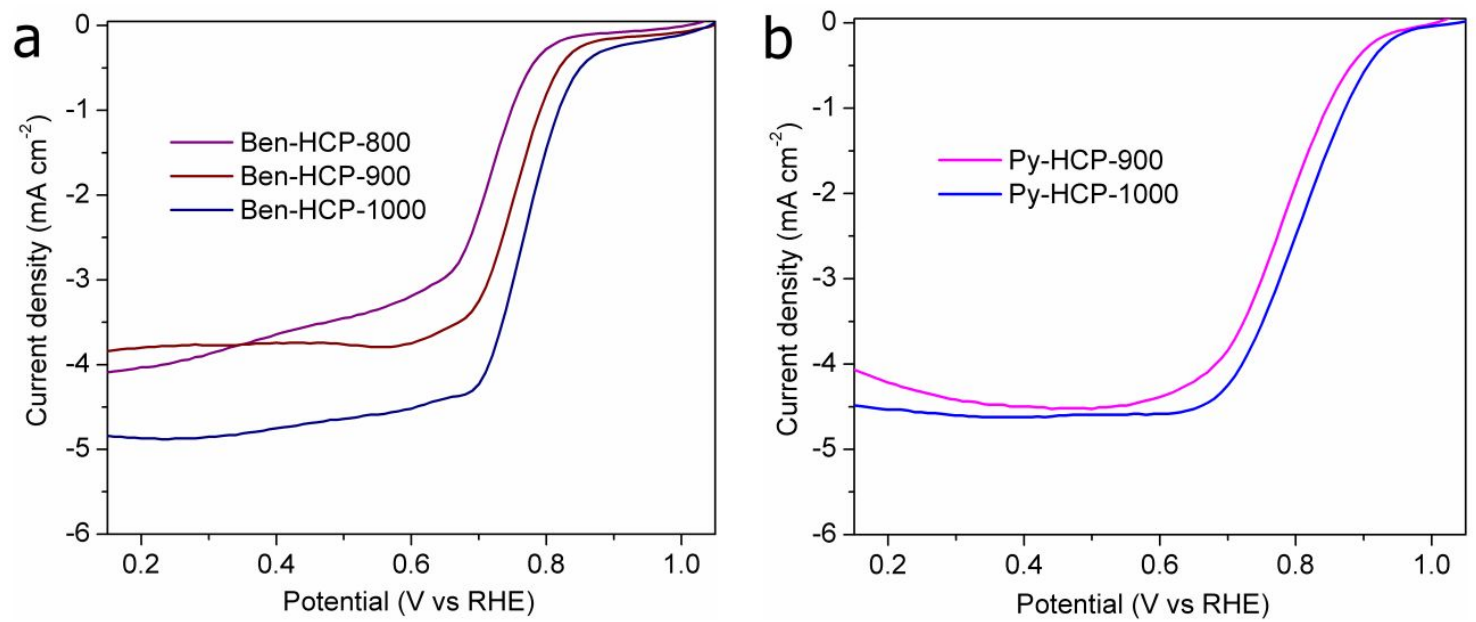

Figure S10. LSV curves of (a) Ben-HCP-x and (b) Py-HCP-x in $\mathrm{O}_{2}$-saturated $0.1 \mathrm{M} \mathrm{KOH}$ electrolyte. 

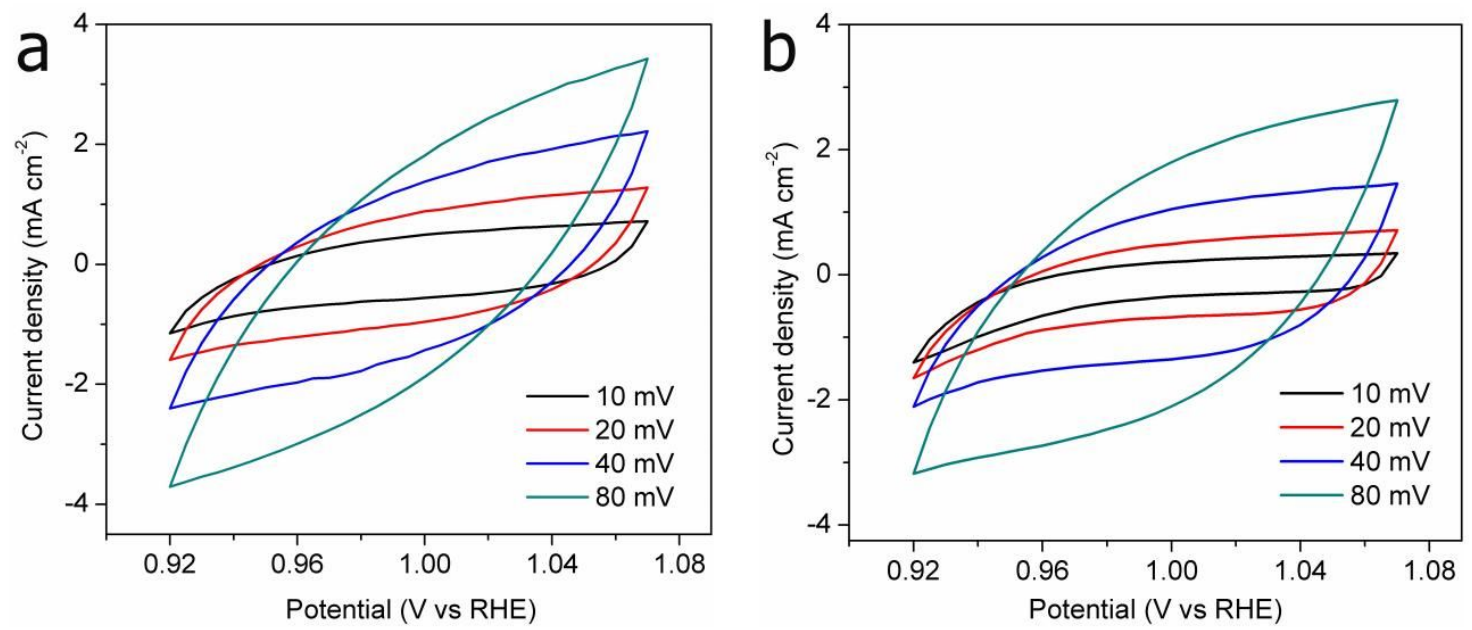

Figure S11. Cyclic voltammograms curves of (a) Ben-NHCP-1000 and (b) Py-NHCP-1000 at different scan rates. 
Table S1. Pore characteristics of Ben-HCP-x, Ben-NHCP-x, Py-HCP-x and Py-NHCP-x.

\begin{tabular}{ccccc}
\hline Sample & $S_{\text {BET }}{ }^{\mathrm{a}}\left(\mathrm{m}^{2} \mathrm{~g}^{-1}\right)$ & $S_{\text {micro }} \mathrm{b}\left(\mathrm{m}^{2} \mathrm{~g}^{-1}\right)$ & $V_{\text {total }}{ }^{\mathrm{c}}\left(\mathrm{cm}^{-3} \mathrm{~g}^{-1}\right)$ & $V_{\text {micro }}\left(\mathrm{cm}^{-3} \mathrm{~g}^{-1}\right)$ \\
\hline Ben-HCP-800 & 498 & 292 & 0.606 & 0.124 \\
Ben-HCP-900 & 460 & 333 & 0.532 & 0.135 \\
Ben-HCP-1000 & 491 & 355 & 0.532 & 0.144 \\
Ben-NHCP-800 & 791 & 281 & 0.958 & 0.125 \\
Ben-NHCP-900 & 824 & 590 & 0.778 & 0.238 \\
Ben-NHCP-1000 & 1165 & 606 & 2.560 & 0.253 \\
Py-HCP-900 & 628 & 277 & 0.498 & 0.121 \\
Py-HCP-1000 & 675 & 432 & 0.491 & 0.177 \\
Py-NHCP-900 & 1122 & 536 & 1.034 & 0.251 \\
Py-NHCP-1000 & 1865 & 1442 & 1.199 & 0.622 \\
\hline
\end{tabular}

a Total surface area calculated using the BET method.

${ }^{b}$ Micropore surface area and volume calculated from t-plot method.

c Total pore volume calculated at $P / P_{0}=0.99$. 
Table S2. N contents (at.\%) of Ben-NHCP-x and Py-NHCP-x evaluated from XPS.

\begin{tabular}{lccccc}
\hline & $\mathrm{N}-1^{\mathrm{a}}$ & $\mathrm{N}-2^{\mathrm{b}}$ & $\mathrm{N}-3^{\mathrm{c}}$ & $\mathrm{N}^{\mathrm{d}}$ & $\mathrm{N}_{\text {total }}$ \\
\hline Ben-NHCP-800 & 1.42 & 0.56 & 1.05 & 0.45 & 3.48 \\
Ben-NHCP-900 & 1.21 & 0.49 & 1.04 & 0.39 & 3.13 \\
Ben-NHCP-1000 & 1.07 & 0.47 & 0.98 & 0.35 & 2.87 \\
Py-NHCP-900 & 1.46 & 0.68 & 0.73 & 0.23 & 3.10 \\
Py-NHCP-1000 & 1.30 & 0.31 & & & \\
\hline
\end{tabular}

${ }^{a}$ pyridinic $\mathrm{N}(\mathrm{N}-1),{ }^{b}$ Pyrrolic N (N-2), ${ }^{\mathrm{c}}$ Graphitic N (N-3), ${ }^{\text {d }}$ Oxidated N (N-4). 
Table S3. Comparison of the ORR catalytic performances in terms of the $E_{\text {onset, }} E_{1 / 2}$ (V vs. RHE) for recently reported metal-free and noble-metal-free electrocatalysts and our optimal $\mathrm{N}$-doped porous carbon catalyst at $1600 \mathrm{rpm}$ in $0.1 \mathrm{M} \mathrm{KOH}$ electrolyte.

\begin{tabular}{|c|c|c|c|c|c|}
\hline & Catalyst & $E_{\text {onset }}$ & $E_{1 / 2}$ & Stability & Reference \\
\hline 1 & $\begin{array}{l}\text { N-doped nanoporous } \\
\text { carbon fiber films }\end{array}$ & 0.97 & 0.82 & - & $\begin{array}{l}\text { Adv. Mater. 2016, 28, } \\
3000\end{array}$ \\
\hline 2 & $\begin{array}{l}\mathrm{N}, \mathrm{P} \text { Co-doped carbon } \\
\text { network }\end{array}$ & $\sim 0.95$ & 0.81 & - & $\begin{array}{l}\text { Angew. Chem. Int. Ed. } \\
\text { 2016, 55, } 2230\end{array}$ \\
\hline 3 & $\mathrm{Fe}-\mathrm{N}_{4}$ & - & 0.90 & $\begin{array}{l}\text { Change in } E_{1 / 2} \text { about } 2 \\
\mathrm{mV} \text { after } 5000 \text { cycles }\end{array}$ & $\begin{array}{l}\text { Angew. Chem., Int. Ed. } \\
\text { 2017, 56, } 6937\end{array}$ \\
\hline 4 & $\begin{array}{l}\text { 3D N-doped carbon } \\
\text { nanotubes }\end{array}$ & 0.91 & 0.84 & $\begin{array}{l}\text { Current retention of } \\
89.7 \% \text { after } 18 \mathrm{~h}\end{array}$ & $\begin{array}{l}\text { Angew. Chem. Int. Ed. } \\
\text { 2017, 56, } 13781\end{array}$ \\
\hline 5 & $\begin{array}{l}\text { Co-N-doped hollow } \\
\text { carbon spheres }\end{array}$ & 0.92 & 0.86 & $\begin{array}{l}\text { Current retention of } \\
93 \% \text { after } 10 \mathrm{~h}\end{array}$ & $\begin{array}{l}\text { Appl. Catal. B-Environ. } \\
\text { 2017, 217, } 477\end{array}$ \\
\hline 6 & $\begin{array}{l}\text { Ultrathin } \\
\text { nitrogen-doped holey } \\
\text { carbon@graphene }\end{array}$ & 1.00 & 0.85 & $\begin{array}{l}\text { No obvious change in } \\
E_{1 / 2} \text { after } 10000 \text { cycles }\end{array}$ & $\begin{array}{l}\text { Angew. Chem. Int. Ed. } \\
\text { 2018, 57, } 16511\end{array}$ \\
\hline 7 & $\begin{array}{l}\text { Iron phthalocyanine } \\
(\mathrm{FePc}) / \mathrm{Ti}_{3} \mathrm{C}_{2} \mathrm{~T}_{\mathrm{x}}\end{array}$ & $\sim 0.93$ & 0.86 & - & $\begin{array}{l}\text { Adv. Mater. 2018, 30, } \\
1803220\end{array}$ \\
\hline 8 & $\begin{array}{l}\mathrm{Fe}-\mathrm{N} \text { doped } \\
\text { mesoporous carbon }\end{array}$ & 0.92 & 0.845 & $\begin{array}{l}\text { No obvious change in } \\
E_{1 / 2} \text { after } 5000 \text { cycles }\end{array}$ & $\begin{array}{l}\text { Chem. Mater. 2019, 31, } \\
3274\end{array}$ \\
\hline 9 & $\mathrm{Fe}-\mathrm{N}_{\mathrm{x}}$ & 0.98 & 0.90 & $\begin{array}{l}\text { Negligible change in } \\
E_{1 / 2} \text { after } 5000 \text { cycles }\end{array}$ & $\begin{array}{l}\text { Nat. Commun. 2019, 10, } \\
1278 .\end{array}$ \\
\hline 10 & $\mathrm{~N}$-doped porous carbon & 0.94 & 0.86 & $\begin{array}{l}\text { Current retention of } \\
95.2 \% \text { after } 50000 \mathrm{~s}\end{array}$ & Nanoscale 2019, 11, 2423 \\
\hline 11 & $\begin{array}{l}\text { Defect-rich and } \\
\text { ultrathin } \mathrm{N} \text { doped } \\
\text { carbon nanosheets }\end{array}$ & 0.95 & 0.82 & $\begin{array}{l}\text { Current retention of } \\
85.6 \% \text { after } 12000 \mathrm{~s}\end{array}$ & $\begin{array}{l}\text { Energy Environ. Sci., } \\
2019,12,322\end{array}$ \\
\hline 12 & $\begin{array}{l}\mathrm{N} \text {-doped carbon } \\
\text { nanotubes }\end{array}$ & 0.88 & 0.82 & $\begin{array}{l}\text { A slight loss of current } \\
\text { retention after } 40000 \mathrm{~s}\end{array}$ & $\begin{array}{l}\text { Nano Energy 2019, 63, } \\
103788\end{array}$ \\
\hline
\end{tabular}




\begin{tabular}{|c|c|c|c|c|c|}
\hline 13 & $\begin{array}{l}\text { Biomass waste-derived } \\
\text { 3D metal-free porous } \\
\text { carbon }\end{array}$ & 1.06 & 0.85 & $\begin{array}{l}\text { Below } 10 \mathrm{mV} \text { change in } \\
E_{1 / 2} \text { after } 5000 \text { cycles }\end{array}$ & $\begin{array}{l}\text { ACS Sustainable Chem. } \\
\text { Eng. } 2019,7,17039\end{array}$ \\
\hline 14 & $\begin{array}{l}\text { Mesopore-dominated } \\
\text { semi-tubular } \\
\text { doped-carbon }\end{array}$ & 1.01 & 0.85 & $\begin{array}{c}\text { Change in } E_{\text {onset }} \text { about } 2 \\
\mathrm{mV} \text { after } 5000 \text { cycles }\end{array}$ & $\begin{array}{l}\text { J. Mater. Chem. A, 2020, } \\
8,9832\end{array}$ \\
\hline 15 & $\mathrm{Fe} / \mathrm{N}-\mathrm{G}-\mathrm{SAC}$ & - & 0.89 & $\begin{array}{l}\text { Change in } E_{\text {onset }} \text { about } 3 \\
\mathrm{mV} \text { after } 10000 \text { cycles }\end{array}$ & $\begin{array}{l}\text { Adv. Mater. 2020, } \\
2004900\end{array}$ \\
\hline 16 & $\begin{array}{l}\text { Single-atom iron } \\
\text { catalysts on } \\
\text { overhang-eave carbon } \\
\text { cages }\end{array}$ & 1.00 & 0.85 & $\begin{array}{l}\text { Current retention of } \\
86.7 \% \text { after } 40000 \mathrm{~s}\end{array}$ & $\begin{array}{l}\text { Angew. Chem. Int. Ed. } \\
\text { 2020, 59, } 7384\end{array}$ \\
\hline 17 & Meso-Fe-N-C & - & 0.846 & $\begin{array}{l}\text { Apparent decay in } E_{\text {onset }} \\
\text { after } 10000 \text { cycles }\end{array}$ & ACS Catal. 2021, 11, 74 \\
\hline 18 & $\begin{array}{l}\text { Fe single atom/cluster } \\
\text { embedded on } \mathrm{N} \text {-doped } \\
\text { carbon }\end{array}$ & 0.97 & 0.898 & $\begin{array}{l}\text { Current retention of } \\
88.5 \% \text { after } 20 \mathrm{~h}\end{array}$ & $\begin{array}{l}\text { Small Methods 2021, } \\
2001165\end{array}$ \\
\hline 19 & $\begin{array}{l}\text { N-doped porous } \\
\text { carbon derived from } \\
\text { HCPs }\end{array}$ & 1.01 & 0.900 & $\begin{array}{l}\text { Current retention of } \\
97.6 \% \text { after } 20000 \mathrm{~s}\end{array}$ & This work \\
\hline
\end{tabular}


Table S4. Comparison of the ORR catalytic performances in terms of $E_{\text {onset, }} E_{1 / 2}(\mathrm{~V}$ vs. RHE) and $J_{L}$ of Ben-HCP-x, Ben-NHCP-x, Py-HCP-x and Py-NHCP-x samples.

\begin{tabular}{|c|c|c|c|}
\hline Catalyst & $E_{\text {onset }}(\mathrm{V}$ vs. RHE) & $E_{1 / 2}(\mathrm{~V} v s . \mathrm{RHE})$ & $J_{L}\left(\mathrm{~mA} \mathrm{~cm}^{-2}\right)$ \\
\hline Ben-HCP-800 & 0.87 & 0.70 & 4.08 \\
\hline Ben-HCP-900 & 0.95 & 0.76 & 3.87 \\
\hline Ben-HCP-1000 & 1.0 & 0.77 & 4.88 \\
\hline Ben-NHCP-800 & 0.95 & 0.811 & 4.18 \\
\hline Ben-NHCP-900 & 0.97 & 0.821 & 4.39 \\
\hline Ben-NHCP-1000 & 1.00 & 0.890 & 5.42 \\
\hline Py-HCP-900 & 0.94 & 0.78 & 4.50 \\
\hline Рy-HCР-1000 & 0.97 & 0.81 & 4.64 \\
\hline Py-NHCP-900 & 1.00 & 0.887 & 5.04 \\
\hline Py-NHCP-1000 & 1.01 & 0.900 & 5.40 \\
\hline
\end{tabular}

